\title{
Lesões Precursoras do Câncer de Colo em Mulheres Adolescentes e Adultas Jovens do Municipio de Rio Branco - Acre
}

\author{
Cervical Cancer Precursor Lesions in Adolescent and Young Adult \\ Women of Rio Branco - Acre \\ Elaine Azevedo Soares Leal ${ }^{1}$, Osvaldo de Sousa Leal Júnior ${ }^{2}$, \\ Maria Helena Guimarães ${ }^{1}$, Maria Nísia Vitoriano ${ }^{1}$, \\ Talita Lima do Nascimento ${ }^{1}$, Olívia Lúcia N. Costa ${ }^{3}$.
}

\begin{abstract}
RESUM0
Objetivo: descrever a freqüência das lesões precursoras do câncer de colo uterino em mulheres de 15 a 29 anos, associando o grau de acometimento com características epidemiológicas e fatores de risco associados.

Métodos: foi realizado estudo transversal com a pesquisa de lesões precursoras do câncer de colo uterino, pelo exame de Papanicolaou, em mulheres de 15 a 29 anos com vida sexual ativa, residentes no municipio de Rio Branco, no periodo de janeiro a setembro de 2001. Como instrumento de investigação, foi aplicada ficha clinico-ginecológica, constando dados epidemiológicos, fatores de risco e resultados do exame fisico-ginecológico, incluindo teste de Schiller e coleta de espécime para exame citopatológico.

Resultados: das 2.397 mulheres estudadas, $155(6,4 \%)$ apresentaram algum tipo de alteração epitelial cervical, sendo 146 (94,2\%) lesões escamosas e 9 (5,8\%) lesões glandulares. Nas mulheres com faixa etária de 15 a 19 anos a freqüência de alteração celular epitelial foi de 6,9\%, semelhante a 6,3\% observada naquelas de 20 a 29 anos (p>0,65). Este tipo de alteração foi associada ao baixo grau de escolaridade ( $p<0,003)$, ao número maior de parceiros $(p<0,04)$, à história de doença sexualmente transmissivel $(p<0,001)$ e ao tabagismo $(p<0,01)$.

Conclusão: a freqüência elevada de lesões precursoras em faixa etária abaixo do esperado, com o padrão epidemiológico observado em outras fases da vida da mulher, evidencia a exposição precoce aos fatores risco, o que antecipa o desenvolvimento do câncer de colo uterino.
\end{abstract}

PALAVRAS-CHAVE: Adolescência. Colo do útero: lesões pré-neoplásicas. Citologia.

\section{Introdução}

O exame de Papanicolaou é utilizado em diversos países para o rastreamento e detecção precoce do câncer de colo uterino. Dada a lenta evolução deste câncer, é possível o diagnóstico na fase intra-epitelial (não-invasiva) em mulheres

${ }^{1}$ Centro de Controle Oncológico do Acre (CECON) da Secretaria de Estado de Saúde e Saneamento do Acre (SESSACRE),

${ }^{2}$ Mestrando do Curso de Pós-Graduação em Medicina e Saúde da UFBA, ${ }^{3}$ Universaidade Federal da Bahia (Salvador, Bahia) Correspondência:

Elaine A. Soares Leal

Alameda Paineras, 239, Chácara Ipê

69911-860 - Rio Branco - Acre

e-mail: ms.elaine@ac.gov.br

Apoio de Financiamento: SESSACRE assintomáticas, quando o tratamento é de baixo custo e tem elevado percentual de cura ${ }^{1}$.

No Brasil, excluindo as causas mal definidas (ou subnotificadas), as neoplasias são o terceiro grupo de doenças mais prevalentes $(11,9 \%)$, ficando atrás apenas das doenças do aparelho circulatório $(27,5 \%)$ e causas externas $(12,6 \%)^{2}$. Entre as mulheres, o câncer de mama é o mais freqüente, seguido pelo câncer de colo uterino, mas este ocupa o primeiro lugar nas regiões Norte e Nordeste do país ${ }^{2}$.

Segundo as estimativas do Ministério da Saúde, para o ano 2001, o câncer de colo uterino ocupa o terceiro lugar em incidência e mortalidade entre as neoplasias no Brasil, sendo o Estado do Acre um daqueles onde esta doença ocupa o primeiro lugar. No ano 2000, o número de casos 
novos diagnosticados no Acre foi de 301 para 18.000 mulheres que se submeteram ao exame, sendo 116 com idade inferior a 30 anos $^{3}$, mas, segundo o Ministério da Saúde, o total esperado seria de 60 casos novos por 100.000 mulheres ${ }^{4}$.

$\mathrm{O}$ número absoluto de casos é maior em mulheres entre 30 e 60 anos. A média de idade de acometimento é de 30 anos em casos de displasia leve ou moderada e de 37 anos quando a displasia é gra$\mathrm{ve}^{5}$. Porém, nos países em desenvolvimento, as mulheres com estas lesões têm idades mais baixas ${ }^{5}$. No Estado do Acre, não existem estudos sobre o tema.

Devido a essas observações realizou-se este estudo, com o objetivo de descrever a freqüência das lesões precursoras do câncer de colo uterino, em mulheres de 15 a 29 anos de idade no município de Rio Branco, associando o grau de acometimento das lesões com características epidemiológicas e fatores conhecidos de risco.

\section{Pacientes e Métodos}

O estudo transversal foi realizado no período de janeiro a setembro de 2001, incluindo mulheres com vida sexual ativa, na faixa etária de 15 a 29 anos, que tiveram o exame de Papanicolaou colhido no Centro de Controle Oncológico do Acre (CECON), de Rio Branco (Acre), da Secretaria de Estado de Saúde e Saneamento. Além desses critérios, as pacientes selecionadas deveriam residir no município de Rio Branco (Acre), não serem referenciadas por outro serviço, não terem diagnóstico prévio de lesão intra-epitelial cervical e concordarem em participar do estudo.

As mulheres da amostra foram selecionadas por meio de livre demanda, esclarecidas quanto à sua participação no estudo e o seu consentimento foi obtido por termo livre e esclarecido, assinado por ela ou o seu responsável legal. As doenças diagnosticadas foram tratadas e acompanhadas no CECON. O estudo foi aprovado pelo Comitê de Ética em Pesquisa da Fundação Hospital Estadual do Acre.

Após a seleção dos casos, foi preenchida a ficha clínico-epidemiológica, contendo dados demográficos (idade, estado civil, escolaridade e ocupação principal), fatores de risco descritos na literatura (idade de início da atividade sexual, número de parceiros sexuais, antecedentes de doença sexualmente transmissivel, paridade, tabagismo, métodos contraceptivos, idade no primeiro parto e resultado de citologia anterior). Foram registrados também os dados de exame físico-ginecológico e dos laudos citopatológicos. De posse do resultado do exame preventivo, as mulheres foram submetidas ao exame ginecológico por médico ginecologista, com utilização do espéculo de Collins para avaliação do canal vaginal e colo, e realização do teste de Schiller.

Os esfregaços do exame citopatológico foram preparados na forma convencional (coloração de Papanicolaou) e avaliados no CECON, e os laudos expressos utilizando a nomenclatura de Bethesda (TBS) ${ }^{6}$. Antes do preparo, as lâminas foram avaliadas quanto à adequação do material. Aquelas consideradas insatisfatórias foram refeitas mediante coleta de nova amostra. Todas as lâminas consideradas como positivas e $10 \%$ das negativas foram reavaliadas por médico citopatologista que desconhecia o resultado anterior.

Os dados foram registrados e analisados por meio do programa SPSS, versão 9.0. Na análise estatística, foram descritas as freqüências das variáveis e a distribuição (média, mediana, moda e desvio-padrão, quando aplicados). A variável-resposta (alteração do epitélio cervical) foi categorizada e estudada em relação à distribuição das variáveis independentes. Nas análises das variáveis qualitativas foi aplicado o teste $\chi^{2}$ ou teste exato de Fisher, conforme a indicação. Na análise das variáveis continuas foi aplicado o teste $t$ de Student. O resultado foi considerado significante se a probabilidade do erro tipo I foi $\leq 5 \%(p<0,05)$. Aqueles resultados associados a variável-resposta com $\mathrm{p} \leq 0,25$ foram incluídos na análise multifatorial, utilizando o teste de regressão logística.

\section{Resultados}

No estudo foram incluídas 2.397 mulheres com idade entre 15 e 29 anos. Dentre estas, predominarem aquelas na faixa etária de 22 a 25 anos (884 mulheres ou $36,9 \%$ ), sendo a média de idade de 23,3 $( \pm 3,6)$ anos. Quanto ao estado civil, 1.351 mulheres $(56,4 \%)$ eram casadas ou tinham companheiro fixo. A maioria (1.228 ou $51,2 \%$ mulheres) tinha escolaridade até o primeiro grau incompleto e 1.341 (55,9\%) eram donas-de-casa. Das 2.397 mulheres estudadas, $1.533(64,0 \%)$ estavam fazendo o exame pela primeira vez e $864(36,0 \%)$ relatavam exame anterior e com resultado normal. No primeiro grupo $(n=1.533)$, a freqüência de lesões foi de $6,3 \%(n=97)$, não havendo diferença estatisticamente significante $(p>0,80)$ da observada nas mulheres $(6,6 \% ; 57 / 864)$ com exame anterior.

$\mathrm{Na}$ Tabela 1, observam-se os dados demográficos das mulheres estudadas, mostrando que a freqüência de lesões do tipo celular epitelial (escamosa ou glandular) do colo uterino foi de $6,4 \%(154 / 2.397)$ e as freqüências foram 
semelhantes $(p>0,64)$ entre as mulheres de 15 a 19 anos $(6,9 \%)$ e de 20 a 29 anos (6,3\%). Essas lesões foram mais freqüentes $(p<0,003)$ nas mulheres de mais baixa escolaridade $(7,8 \%)$ do que naquelas de maior escolaridade $(4,8 \%)$. A ocupação "dona-de-casa" e o estado civil "casado ou com companheiro fixo" não foram associados (respectivamente, $p>0,70$ e $p>0,16$ ) àquelas lesões.

Tabela 1 - Dados demográficos e indicadores sociais em 2.397 mulheres de 15 a 29 anos, com alteração celular epitelial (escamosa ou glandular), avaliada pelo exame citológico de Papanicolaou, Rio Branco (AC).

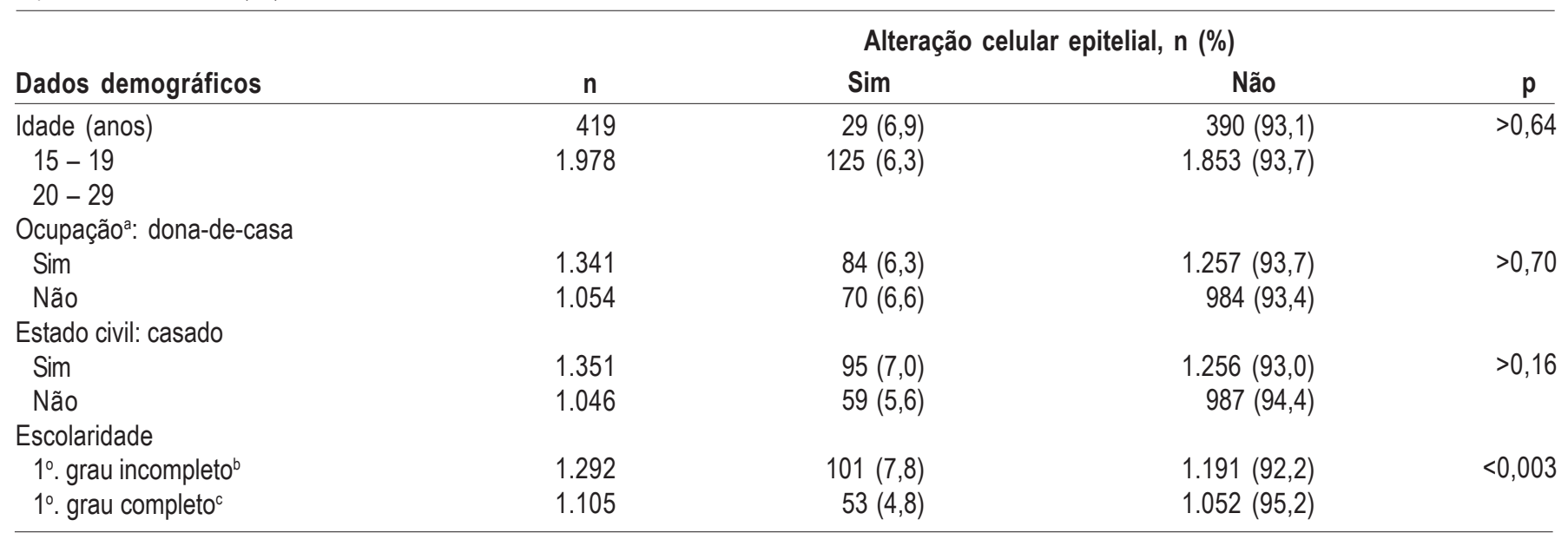

a 2 mulheres não foram incluídas; ${ }^{\mathrm{b}}$ incluindo também analfabetas ou de menor escolaridade; ${ }^{\mathrm{c}}$ ou maior escolaridade.

Quanto aos antecedentes ginecológicos (Tabela 2), a presença de lesões do tipo celular epitelial foi significativamente $(p<0,04)$ associada ao número de parceiros, porque as mulheres com parceiro único tiveram freqüência inferior de lesões $(4,9 \%)$ ao serem comparadas $\left(\chi^{2}=6,32\right.$; $\mathrm{p}<0,02)$ com aquelas com 2 ou mais parceiros $(8,1 \%)$. Os demais antecedentes - época da menarca ( $p>0,56)$, do $1^{\circ}$ coito $(p>0,20)$, número de filhos $(p>0,09)$ e idade no $1^{\circ}$ parto $(p>0,22)$ - não foram associados, estatisticamente, à presença de lesões. Quanto ao tipo de relacionamento se- xual, entre aquelas com história de coito anal $(19,5 \% ; n=467)$, a freqüência de lesões foi de $7,9 \%$ $(n=37)$, não sendo estatisticamente diferente $(p>0,16)$ da observada nas mulheres 114/1.862 (6,1\%;) sem a mesma história. A Tabela 3 mostra que a história de DST $(1.4 / 76(18,4 \%, \mathrm{p}<0,00002)$ e de tabagismo $(40 / 44(9,1 \%), p<0,01)$ foi significativamente associada à presença de lesões do colo uterino, porém, a mesma associação não foi observada com o uso de algum método contraceptivo $(\mathrm{p}>0,80)$.

Tabela 2 - Antecedentes ginecológicos de 2.397 mulheres associados à presença de alteração celular epitelial (escamosa ou glandular), Rio Branco (AC).

\begin{tabular}{|c|c|c|c|c|}
\hline Antecedentes ginecológicos & $\mathrm{n}$ & Sim & Não & $p$ \\
\hline \multicolumn{5}{|l|}{ Menarca (anos) a } \\
\hline $13-15$ & 2.220 & $145(6,5)$ & $2.075(3,5)$ & \\
\hline$\geq 16$ & 96 & $5(5,2)$ & $91(94,8)$ & \\
\hline \multicolumn{5}{|l|}{$1^{0}$ coito (idade, anos) ${ }^{b}$} \\
\hline$\geq 16$ & 1.224 & $69(5,6)$ & $1.155(94,4)$ & \\
\hline \multicolumn{5}{|l|}{$\mathrm{N}^{0}$. parceiros } \\
\hline 1 & 978 & $48(4,9)^{d}$ & $930(95,1)$ & $<0,04$ \\
\hline $2-4$ & 967 & $76(7,9)^{\mathrm{e}}$ & $891(92,1)$ & \\
\hline $5-7$ & 240 & $13(5,4)^{f}$ & $227(94,6)$ & \\
\hline$\geq 20$ & 454 & $22(4,8)$ & $432(95,2)$ & \\
\hline
\end{tabular}

2.397 mulheres; ${ }^{\text {b }} 2.383$ casos; ${ }^{c} 1.837$ mulheres. $d$ vs e, $f, g: \chi^{2}=6,32 ; p<0,20$ 
Tabela 3 - Fatores de risco descritos como associados às alterações celulares epiteliais (escamosa ou glandular), observadas em mulheres de Rio Branco (AC).

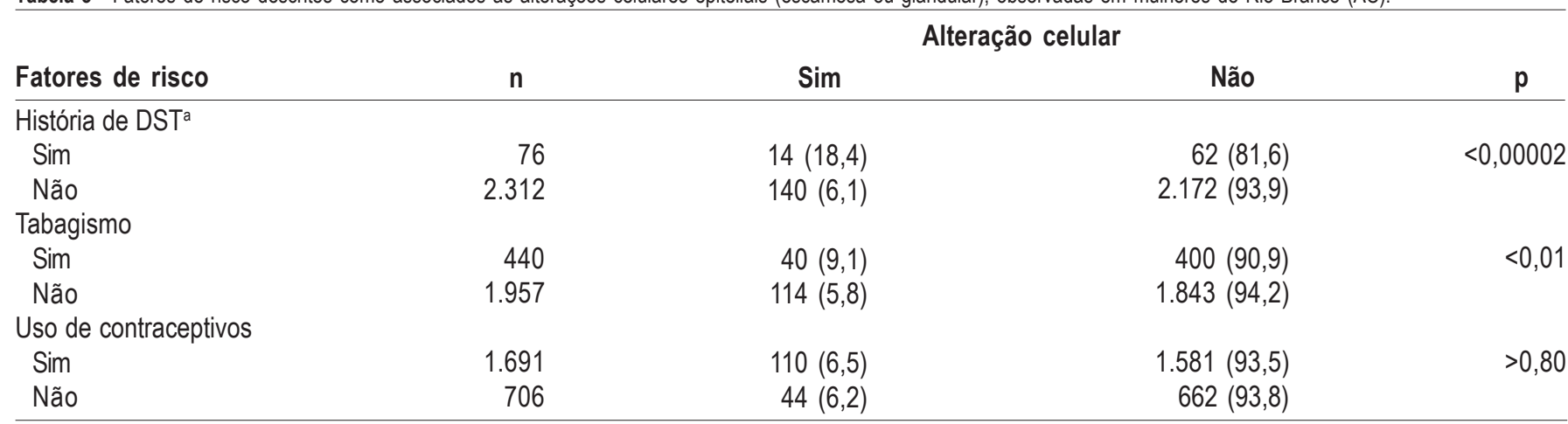

${ }^{a} n=2.388$

Nas 2.397 mulheres estudadas, a maioria $(\mathrm{n}=1.691 ; 70,5 \%)$ utilizava algum método contraceptivo, com a seguinte distribuição: $1.112(46,4 \%)$ utilizavam métodos hormonais (oral ou injetável); $342(14,3 \%)$ tinham parceiro que usava condom; 6 $(0,3 \%)$ usavam DIU e $244(10,2 \%)$ foram laqueadas. Apenas $11(0,6 \%)$ mulheres usavam dois métodos contraceptivos e uma fazia uso de três métodos.

O exame ginecológico constatou: secreção vaginal anormal em 93,4\% ( $n=2.239)$; teste de Schiller positivo em $62,4 \%(n=1.496)$; lesão de epitélio cervical em $24,2 \%(n=581)$ e lesão sugestiva de DST em apenas 1,7\% $(n=40)$ dos casos. Nesses casos, as freqüências de lesão celular epitelial foram, respectivamente, de $6,2 \%(n=139, p>0,09)$, $7,6 \%(n=114, p<0,002), 9,0 \%(n=51, p<0,008)$ e $5,0 \%$ $(\mathrm{n}=2, \mathrm{p}>0,71)$.

Com essas análises univariadas foi aplicada a análise multifatorial de regressão logística, incluindo as variáveis associadas $(\mathrm{p} \leq 0,25$; $\mathrm{IC}=95 \%$ ), à variável dependente (presença de lesão do epitélio cervical), obtendo-se os seguintes resultados: (i) quanto ao estado civil, ter companheiro fixo apresenta risco de $58 \%$ de desenvolver alteração celular cervical, $\mathrm{OR}=1,58(1,04-2,40)$, $\mathrm{p}<0,03$; (ii) escolaridade até o primeiro grau incompleto tem risco de $48 \%$, OR $=1,48(0,98-2,25)$, mas sem alcançar significância estatística ( $\mathrm{p}>0,06)$; (iii) com história passada de DST, o risco foi de $176 \%$, OR = 2,88 (1,34-6,17), $\mathrm{p}<0,007$; (iv) quanto ao tabagismo, o risco foi de $50 \%$, OR $=1,50$ (0,96-2,32), mas sem alcançar significância estatística ( $p>0,07)$; (v) para teste de Schiller positivo, o risco foi de $73 \%$, com OR $=1,73(1,11-2,70), p<0,02$.

\section{Discussão}

Dentre todas as localizações, o câncer de colo do útero é o que apresenta um dos mais altos potenciais de prevenção e cura, sendo este próximo a $100 \%$, quando diagnosticado precocemente ${ }^{7}$. A incidência máxima dessa neoplasia situa-se entre 40 e 60 anos de idade, e apenas uma pequena porcentagem ocorre antes dos $30 \operatorname{anos}^{8}$. Embora o Brasil tenha sido um dos primeiros países do mundo a introduzir o exame de Papanicolaou para a detecção precoce do câncer de colo, a doença continua a ser um grave problema de saúde pública. Isto porque apenas $30 \%$ das mulheres submetemse ao exame citopatológico pelo menos três vezes na vida, o que resulta em diagnósticos já em fase avançada em $70 \%$ dos $\operatorname{casos}^{7}$.

No presente estudo, foi observada freqüência elevada $(6,4 \%)$ de lesões precursoras do câncer do colo uterino em mulheres com a faixa etária inferior a esperada para esta doença, principalmente adolescentes que aparentam ser mais predispostas aos riscos associados ao câncer uterino. Segundo Mangan et al. ${ }^{9}$, as adolescentes são mais vulneráveis aos fatores de risco, por apresentarem a zona de transformação do colo localizada na ectocérvice, estando assim exposta aos agentes potencialmente associados da neoplasia, tais como: múltiplos parceiros sexuais, o não-uso dos métodos de barreira para a contracepção e prevenção de doenças sexualmente transmissiveis.

Estudos sugerem que o risco de neoplasia está relacionado à idade da primeira relação sexual e múltiplos parceiros, indicando que o coito precoce pode aumentar a sensibilidade aos efeitos de um agente sexual transmitido ${ }^{10}$. Esta constatação é sustentada por outras evidências, as quais mostram que o intervalo entre a menarca e o primeiro coito parece ser mais relevante que a idade da primeira relação ou a idade das primeiras relações regulares, ligando, assim, o risco de neoplasia à idade "sexual" mais do que à cronológica ${ }^{10}$.

Ao relacionar a idade de início da atividade sexual e a presença de lesões precursoras, não observamos diferença significante. Mas, por ser um fator de risco comprovadamente estudado, vale ressaltar a freqüência de $7,2 \%$ de mulheres que se iniciaram sexualmente antes dos 15 anos, al- 
gumas antes mesmo da menarca. Quanto ao número de parceiros, observou-se a tendência do aumento da freqüência de alteração celular epitelial, com aumento do seu número.

No Brasil, a Pesquisa Nacional sobre Saúde e Demografia, realizada pela Sociedade Civil BemEstar Familiar (BEMFAM), em 1996, demonstrou que, nos últimos 10 anos, a fecundidade das mulheres tem diminuído em torno de $30 \%$ em todas as faixas etárias, com exceção da faixa adolescente. No ano de 1999, no Brasil, houve 705.246 partos de adolescentes, sendo $5.521(0,8 \%)$ no Acre $^{11}$, mas em 2000 pariram em Rio Branco (AC), em uma única maternidade, 1.059 adolescentes, correspondendo a $29,8 \%$ dos 3.552 partos. Também, no presente estudo foi demonstrado que a média de idade no primeiro parto é de 17,9 anos.

Foi observada forte associação entre a presença de alteração celular epitelial e a escolaridade até o primeiro grau incompleto, o que pode ser justificado pela falta de conhecimento quanto ao exame de Papanicolaou e os beneficios de fazêlo rotineiramente, além de outros fatores de risco associados ao câncer de colo uterino, no grupo de pessoas com baixos indicadores de desenvolvimento humano. Estudos realizados em Santiago (Chile) ${ }^{12}$ e na Cidade do México ${ }^{13}$, por exemplo, mostraram que a deficiência do conhecimento do exame de Papanicolaou também é componente freqüente em mulheres com baixa escolaridade.

As mulheres com história de DST tiveram mais freqüentemente alterações epiteliais, assim como aquelas com o hábito de fumar. O uso de contraceptivos, embora conhecido como fator de risco associado ao câncer de colo, neste estudo não mostrou correlação, mas houve maior freqüência de lesões celulares em mulheres usuárias de contraceptivos hormonais.

$\mathrm{Na}$ atualidade, a infecção pelo papilomavirus humano reveste-se de grande relevância, por ser agente potencialmente desencadeador de neoplasias intra-epiteliais e invasivas do trato genital inferior da mulher. Fatores de risco para esta infecção estão presentes em jovens com atividade sexual, maior número de parceiros, com algum grau de imunossupressão, expostas a outras DSTs, fumantes e usuárias de anticoncepcionais hormonais ${ }^{14,15}$.

Como descrito, fumar é outro fator de risco para câncer de colo $^{16}$, mas um estudo realizado por Cuzick et al. ${ }^{17}$, não observou essa associação em mulheres jovens. O epitélio cervical das fumantes tem número menor de células de Langerhans do que as não-fumantes ${ }^{15}$, facilitando as lesões virais, que seriam o primeiro passo no processo de carcinogênese, que de outra maneira necessitaria de tempo mais longo para ter impacto sobre o risco de câncer de colo uterino.
Em conclusão, este trabalho, além de encontrar resultados semelhantes aos observados na literatura sobre os fatores associados ao câncer de colo uterino, chama atenção, fundamentalmente, para o fato de que as jovens acreanas estão expostas aos fatores de risco para esse câncer e suas lesões precursoras, e em nada diferem das jovens de outras regiões do Brasil. A freqüência de lesões celulares é elevada, mostrando a necessidade de programa de atenção específico para mulheres adolescentes, porque, do contrário, espera-se o aumento progressivo de casos de câncer de colo uterino, com grande impacto médico-social.

\section{ABSTRACT}

Purpose: to describe the frequency of precursor lesions of cervical cancer in 15 to 29-year-old women, associating the degree of damage with the epidemiologic characteristics and associated risk factors.

Methods: a transverse study was performed, where the precursor lesions of cervical cancer were investigated through Papanicolaou test in 15 to 29 year-old women with active sexual life, living in Rio Branco (AC), in the period from January to September 2001. The investigated data included epidemiologic information, risk factors and physical-ginecological examination results, including Schiller test and smears for cytopathologic test.

Results: of the 2,397 women studied, 155 (6.4\%) showed some kind of cellular epithelial alteration, 146 (94.2\%) squamous lesions and $9(5.8 \%)$ glandular lesions. In 15 to 19 year old women, the frequency (6.9\%) of cellular epithelial alteration was similar to that observed in 20 to 29-year-old women $(p>0.65)$. These alterations were associated with low educational level $(p<0.003)$, with the number of sexual partners $(p<0.04)$, with STD history $(p<0.001)$ and smoking habits $(p<0.01)$.

Conclusions: the high frequency of precursor lesions in an age lower than expected, and following an epidemiologic pattern observed in other phases of women's life, shows the early exposure to risk factors, which anticipates the development of cervical cancer.

KEYWORDS: Adolescent. Uterine cervix. Cervical cancer. Papanicolaou. NIC.

\section{Agradecimentos}

A Sra. Rosário Noronha, médicos (as) e funcionários (as) do CECON (AC), pela presteza e atenção que nos dispensaram, e ao Prof. José Tavares-Neto, pela excepcional ajuda em cada fase deste trabalho. 


\section{Referências}

1. Brenna SMF, Hardy EE, Zeferino LC, Namura I. Conhecimento, atitude e prática do exame de Papanicolaou em mulheres com câncer de colo uterino. Cad Saúde Pública 2001; 17:909-14.

2. OPAS. Organización Panamericana de La Salud. La salud en las Americas. Geneva; 1998. p.125-36.

3. Secretaria de Estado de Saúde e Saneamento do Acre. CECON. Centro de Controle Oncológico do Acre. Relatório geral. Rio Branco; 2000. p.20.

4. Ministério da Saúde. Estimativas da incidência e mortalidade por câncer no Brasil. Brasília; 2000. p.60.

5. Hossfeld DK, Sherman CD, Love RD, Bosch FX. Manual de oncologia clínica. $6^{\mathrm{a}}$ ed. São Paulo: Fundação Oncocentro; 1999. p.399.

6. Solomon D, Darvey D, Kurman R, et al. The 2001 Bethesda System: terminology for reporting results of cervical cytology. JAMA 2001; 287:2114-9.

7. INCA. Instituto Nacional do Câncer. Programa Nacional de Controle do Câncer do Colo do Útero e de Mama - Viva Mulher. Prevenção e Detecção. Disponivel em: http://www.inca.org.br. Acesso em: 07 nov 2001.

8. Ministério da Saúde. Normas e Recomendações do Instituto Nacional do Câncer/MS. Recomendações básicas para o controle do câncer do colo do útero no Brasil. Rev Bras Cancerol 2000; 46:23-33.

9. Mangan SA, Legano LA, Rosen CM, et al. Increased prevalence of abnormal Papanicolaou smears in urban adolescents. Arch Pediatr Adolesc Med 1997; 151:481-4.
10.Brito NMB, Moreira SFS, Ferreira MA, Lopes RV, Bastos AAC. Aspectos epidemiológicos das neoplasias intraepiteliais cervicais identificadas por citologia oncótica. Rev Para Med 2000; 14:42-6.

11. Costa MCO, Santos CAT, Sobrinho CL, Freitas JO, Ferreira KASL. Indicadores materno-infantis na adolescência e juventude: sociodemográfico, prénatal, parto e nascido-vivos. J Pediatr (RJ) 2001; 77:235-42.

12.Ministério da Saúde. Área de Saúde do Adolescente e do Jovem. Gravidez na adolescência, uma questão de Saúde Pública. J FEBRASGO 2000; 3:7-10.

13.Lamadrid Alvarez S. Aspectos socio-culturales de la sexualidad como factores obstaculizantes de la prevención secundaria del cáncer cérvico uterino. Cad Saúde Pública 1998; 14 (Supl 1):33-40.

14.Najera Aguilar P, Lazcano Ponce EC, de Ruiz PA, Ramirez Sanchez T, Cantoral Uriza L, Hernandez Avila M. Factors associated with Mexican women's familiarity with the purpose of the Pap test. Bull Pan Am Health Organ 1996; 30:348-53.

15.Josefsson AM, Magnusson PK, Ylitalo N, et al. Viral load of human papilloma virus 16 as a determinant for development of cervical carcinoma in situ: a nested case-control study. Lancet 2000; 355:2189-93.

16.Parazzini F, Chatenoud L, La Vecchia C, Negri E, Franceschi S, Bolis G. Determinants of risk of invasive cervical cancer in young women. Br J Cancer 1998; 77:838-41

17.Cuzick J, Sasieni P, Singer A. Risk factors for invasive cervix cancer in young women. Eur J Cancer 1996; 32A:836-41.

\section{COMUNICADO AOS ASSOCIADOS E LEITORES}

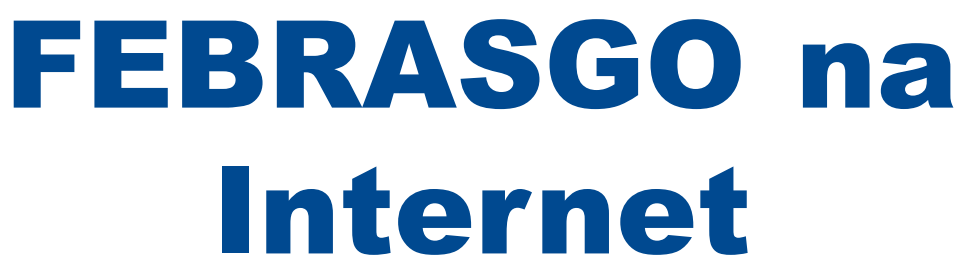

Para maiores informações temos à disposição quatro endereços eletrônicos:

febrasgopresiden@uol.com.br secretaria_executiva@febrasgo.org.br publicacoes@febrasgo.org.br tego_habilitacao@febrasgo.org.br 